Revue européenne des migrations internationales

vol. $19-n^{\circ} 2$ | 2003

Les initiatives de l'étranger et les nouveaux

cosmopolitismes

\title{
Communautés communicantes : étude de quatre radios à Marseille
}

\section{Katrine Rømhild Benkaaba}

\section{(2) OpenEdition}

Journals

Édition électronique

URL : https://journals.openedition.org/remi/2957

DOI : $10.4000 /$ remi.2957

ISSN : $1777-5418$

Éditeur

Université de Poitiers

Édition imprimée

Date de publication : 1 juillet 2003

Pagination : 127-140

ISBN : 2-911627-34-2

ISSN : 0765-0752

Référence électronique

Katrine Rømhild Benkaaba, "Communautés communicantes : étude de quatre radios à Marseille », Revue européenne des migrations internationales [En ligne], vol. 19 - n² | 2003, mis en ligne le 19 avril 2007, consulté le 14 avril 2022. URL : http://journals.openedition.org/remi/2957 ; DOI : https://doi.org/ 10.4000/remi.2957

Ce document a été généré automatiquement le 14 avril 2022.

(c) Université de Poitiers 


\title{
Communautés communicantes : étude de quatre radios à Marseille
}

\author{
Katrine Rømhild Benkaaba
}

1 Les radios dites «communautaires" constituent un champ social de communication qui nous ouvre une perspective interne sur l'attribution dynamique de sens à la notion de «communauté». À travers une étude qualitative de quatre radios marseillaises, nous avons analysé cette communication qui n'a pas trait à la prééminence de l'affirmation d'une identité collective culturelle par rapport à un "autre", mais qui s'oriente vers les membres supposés d'une même communauté.

2 Toute identité implique des processus de construction et de maintien de «frontières ». Celles-ci peuvent cependant être construites de manières diverses, et n'impliquent pas obligatoirement les notions d'antagonisme et d'enfermement. Dans le cas des quatre radios, la communication d'identité collective culturelle est caractérisée par une polysémie qui transforme les pôles - souvent présupposés d'opposition - de "communauté ethnique» et de «citoyenneté républicaine» en ressources complémentaires dans la construction sociale d'identités hybrides et souples.

3 L'étude est basée sur une enquête de terrain conduite à Marseille entre le 10 janvier et le 30 juin 2000. Le choix de la problématique a été dicté avant tout par le souhait d'étudier simultanément plusieurs groupes culturels à l'intérieur d'un même espace social et géographique. Les questions relatives à l'identité culturelle collective étant en quelque sorte la raison d'être des radios « communautaires ", elles présentent en outre l'avantage en tant qu'objet d'étude d'être analytiquement délimitables et accessibles sur le plan pratique. Le choix définitif de ces quatre radios se fonde non seulement sur la dimension explicitement « communautaire » de leurs émissions mais aussi sur leur ancrage local ${ }^{1}$.

Quatre radios marseillaises

4 Les quatre radios en question sont "Radio Gazelle », « Radio Galère ", «Radio Diva » et «Radio JM ». Elles ont toutes été fondées au début des années quatre-vingts avec la libéralisation des ondes et le changement de la loi 1901 qui réinstaurait le droit d'association des étrangers en 1981. Ces changements ont permis la légalisation d'un 
phénomène qui se développait depuis plusieurs années sous une forme illégale ${ }^{2}$. Aujourd'hui les radios ont plus de vingt ans et sont solidement ancrées dans le paysage radiophonique marseillais.

5 Radio Gazelle se présente comme la radio «multi-communautaire » de Marseille. Ses émissions s'orientent en grande partie vers un public arabophone et musulman, et, dans une moindre mesure vers un public d'origine africaine, sud-américaine, caraïbe et asiatique. Durant l'enquête, les émissions à caractère arabo-musulman étaient regroupées les jours de semaine et les autres émissions concentrées le week-end. Cinq fois par jour Radio Gazelle transmet l'appel à la prière musulmane.

6 Radio Galère se définit dans sa charte comme "authentiquement associatif, libre, indépendant et interculturel ». Chaque semaine une vingtaine d'heures sont consacrées à des émissions bilingues qui concernent l'Algérie, la Grèce, les Balkans, l'Océan Indien, les Antilles, la Kabylie, l'Arménie, l'Amérique du Sud, La Réunion, Le Cap-Vert, les Comores, la Corse et l'Italie.

7 Les émissions de Radio JM (Juive de Marseille) varient entre musique, discussions, actualités et reportages sur Marseille et Israël. Le vendredi est consacré à une série d'émissions à caractère religieux qui préparent le Shabbat. Radio JM collabore avec Radio Shalom, basée à Paris, notamment pour ce qui concerne les informations et reportages sur Israël.

8 Radio Diva est la radio italienne de Marseille, ce qui s'exprime à travers ses émissions bilingues franco-italiennes, et sa musique italienne. Orientée principalement vers un public âgé, la radio est également le forum d'autres formes de sociabilité que la communication radiophonique, comme des soirées dansantes et des voyages organisés en Italie et en Espagne.

Réflexions méthodologiques

9 Les radios forment un espace social particulier dont les caractéristiques ont des conséquences pour l'approche méthodologique. Elles sont le cadre d'une pratique communicative plutôt que d'une interaction physique. De plus il s'agit d'une communication fugitive en comparaison, par exemple, avec celle des médias écrits. Elle passe par les paroles et par les sons - notamment la musique ${ }^{3}$.

Les méthodes utilisées ont été les entretiens qualitatifs, l'observation participante dans les studios et l'enregistrement et la transcription de nombreuses émissions. Les différents outils et réflexions méthodologiques éclairent des aspects complémentaires de la problématique.

11 Les entretiens ont été menés avec 33 animateurs et 15 auditeurs. Le choix de l'entretien ouvert semi-structuré, basé sur un guide préalablement élaboré, a permis de saisir des aspects imprévus de la problématique. Afin d'attribuer une dimension dynamique aux données, chaque informateur a été interviewé au moins deux fois. La majorité des entretiens ont ensuite été transcrits avant de faire l'objet d'une analyse codée.

12 Une observation participante a été menée dans les studios des quatre radios. ${ }^{4}$. Il s'agissait de visites de quelques heures à des moments variables; ce qui a permis de couvrir la majorité des émissions de Radio Gazelle et des émissions bilingues de Radio Galère, ainsi que certaines émissions de Radio JM et Radio Diva. Bien que l'observation ait été plus silencieuse que participante durant les passages du personnel à l'antenne, les pauses, les attentes et les diffusions musicales ont largement permis une interaction avec les animateurs et une interrogation sur la pratique en cours. 
13 L'observation participante dans les studios a permis de découvrir le montage et la programmation des différentes émissions - les choix rédactionnels, les commentaires et points de vue des animateurs, des appels d'auditeurs qui ne passent pas à l'antenne etc. En outre, la présence continue dans les locaux à favoriser l'établissement de contacts avec de nouveaux informateurs.

14 La communication radiophonique permet une autre sorte « d'observation » qui est celle de l'écoute. Il est, en effet, possible d'accéder à la radio un peu partout - sur un baladeur, à la maison - ce qui permet un contact permanent et une connaissance de chaque station. Nous avons pu ainsi enregistré 36 émissions, que nous avons ensuite analysées. Nous avons aussi retranscris entièrement des passages parlés sélectionnés qui ont fait l'objet d'un traitement en profondeur.

15 Les études récentes des mass média réservent, quelle que soit leur approche, une place importante à la réception et à l'utilisation de la communication (Hall et al., 1980 ; Lull, 1995). Cependant, il s'est avéré plus difficile que prévu d'établir un contact, à partir des radios, avec les auditeurs. Dans sa globalité, les données analysées nous informent donc plus sur la nature de la communication des radios que sur les processus de réception et d'interprétation chez les auditeurs. Cependant, cette dernière dimension n'est pas absente de nos analyses notamment à travers leurs nombreux appels durant les émissions. Nous avons aussi réalisé une quinzaine d'entretiens avec des auditeurs (dont quatre de Radio Galère, cinq de Radio Gazelle, quatre de radio Diva et deux de Radio $\mathrm{JM})^{5}$.

Communauté, communautaire...

16 La notion de "communauté » se trouve au cœur de notre problématique. En tant que «signifiant» relié à des "signifiés", elle fait partie de l'objet d'étude. En effet, l'enquête menée avait pour objectif d'identifier les modalités de l'attribution de sens à cette notion dans les différentes émissions. Il faut donc distinguer l'utilisation que les animateurs et auditeurs des quatre radios font de cette notion de celle qui est sousjacente à notre analyse.

17 Les radios en question n'existent pas dans un huis clos sémantique, mais doivent être comprises par rapport à un contexte plus large. Bien que le «modèle républicain » français ne permette pas la distinction formelle des citoyens à partir des caractéristiques culturelles ou cultuelles - particularité française qui entretient un certain niveau de suspicion à l'égard de la notion de " communauté » -, de nombreux discours politiques, médiatiques et populaires n'ont de cesse de consacrer des « communautés » présentées comme naturellement délimitées. La référence justifiant cette classification varie - les personnes perçues ou se présentant comme membres d'une même "communauté » peuvent par exemple partager une origine nationale (algérienne, chinoise...) ou régionale («maghrébine», «asiatique »), certaines caractéristiques culturelles (kabyles, juifs sépharades) ou une religion (musulmans, juifs...). La " communauté » a parfois un fonctionnement sémantique proche du terme classificateur "race", toujours en vigueur dans les pays anglophones, mais dans le contexte français elle renvoie à un plus grand éventail d'interprétations possibles. Ainsi le terme existe également comme concept juridique - la «communauté urbaine » par exemple - et il peut être utilisé de manières très différentes.

18 Dans le cas des radios "communautaires", la référence semble à première vue largement basée sur les premiers critères mentionnés - culturels ou cultuels. 

de repère terminologique pour cette utilisation. Le texte de Barth (1969) a introduit les frontières comme métaphore analytique centrale pour les études relatives à l'ethnicité. Cette métaphore met l'accent sur les processus à travers lesquels les différences culturelles deviennent socialement significatives plutôt que sur une essence statique culturelle que chaque groupe possèderait. La métaphore de la frontière ne suffit pas, cependant, à rendre la notion d'ethnicité opératoire pour l'analyse concrète. Toute sorte d'identité implique la création de frontières et d'oppositions (Mouffe, 1993). Il existe de nombreuses formes d'identité collective culturelle. Pour distinguer la particularité de l'identité ethnique il faut rappeler que celle-ci se construit autour de l'idée d'une origine commune et d'un héritage culturel. Par rapport aux quatre radios étudiées il est donc pertinent de se demander dans quelle mesure la "communauté " renvoie à une notion implicite d'ethnicité.

Dans le prolongement de cette interrogation se trouve celle de la nature des frontières socialement construites et de l'emprise des différenciations qu'elles véhiculent. Comme l'affirme Wicker (1997 : 24), les stéréotypes que l'on retrouve dans une publicité ou une dispute maritale ont peu en commun avec ceux qui légitiment les guerres civiles, même s'il s'agit fondamentalement des mêmes mécanismes. Il est donc nécessaire de distinguer s'il s'agit d'une frontière absolue marquée par l'antagonisme social ou bien d'une frontière souple et potentiellement ouverte. En d'autres termes: la dimension « communautaire » des radios véhicule-t-elle une fermeture identitaire?

21 La perspective des acteurs constitue un autre paramètre analytique intéressant. Les radios forment le cadre d'une communication « interne » dans la mesure où il s'agit de la construction continue de sens d'un «nous " perçu en termes de « communauté » et non pas en termes d'interaction ou d'opposition avec un « eux » extérieur. Elles nous permettent donc d'assister à un processus où le sens attribué à la notion de « communauté » émane d'acteurs qui se définissent en termes d'identité commune. La distinction interne-externe ${ }^{6}$ sous-entendue par ces remarques est d'ordre analytique puisque c'est justement la nature instable et socialement construite de la frontière entre « eux » et « nous » qui est au centre du questionnement.

Les travaux de Benedict Anderson (1983) sur l'origine du nationalisme présente une définition intéressante du terme "communauté ». Il dit que toute communauté plus large que le village où tout le monde se connaît est en effet "imaginée " sans qu'elle soit moins « réelle » dans son fonctionnement pour autant. La définition d'Anderson reflète bien le déplacement théorique passant de l'intérêt pour les relations sociales à celui de l'imagination et de la construction sociale. Il a été remarqué cependant, à juste titre, qu'Anderson ne parvenait pas à expliquer pourquoi cette abstraction imaginée arrivait à mobiliser tant de personnes, dont certaines prêtes à se sacrifier pour la «communauté » (Amit, 2002). Cette force mobilisatrice ne peut s'expliquer, selon Hertzfeld (1997:6), si l'on ne tient pas compte des détails empiriques de la vie quotidienne - la famille, l'amitié, tout ce par rapport à quoi la réalité est interprétée. Riche de ces critiques, l'approche d'Anderson a toujours le mérite d'ouvrir un répertoire analytique fructueux qui nous permet dans l'analyse de la communication radiophonique de nous concentrer sur les manières dont la «communauté » est imaginée.

Quels sont les rapports entre « communauté » et langue ; « communauté » et musique ? Dans l'analyse, nous allons distinguer trois aspects différents de la langue parlée telle 
qu'elle apparaît dans les émissions : la langue comme compétence sociale, la langue comme moyen de participation et la langue en tant que discours. Nous allons analyser séparément la dimension musicale même si les deux formes d'expression, langue et musique, sont en réalité liées entre elles.

Bilinguisme et identité

Les échanges linguistiques et les énoncés ne transmettent pas uniquement un contenu sémantique, ils exposent aussi une compétence sociale (Bourdieu, 1982). Dans ce sens, les caractéristiques phoniques linguistiques font partie de la construction sociale de l'identité individuelle et collective. Fishman (1989) montre ainsi que la compétence linguistique peut être interprétée comme faisant partie du " patrimoine » ethnique.

le cas de radios "Gazelle ", "Galère " et "Diva ", qui se caractérisent par des émissions bilingues, on peut observer la même manière d'organiser le bilinguisme.

« (Italien :) Buonasera, (français :) bonsoir, [en italien : présentation du programme du soir, invitation aux auditeurs à appeler], (Français :) oui, n'hésitez pas à nous appeler pour une dédicace, une chanson [en italien : quelle belle soirée, ça me rappelle (...)] (Français :) et maintenant, pour commencer, cette belle chanson que vous connaissez tous : (...) » (émission, Radio Diva).

L'animateur oscille entre les deux langues; les premières informations prononcées en italien ne sont pas répétées en français, mais l'invitation aux auditeurs à téléphoner est lancée dans les deux langues. La dernière phrase commence en italien et finit en français. Ainsi, le principe de l'alternance affecte non seulement la totalité de la communication mais aussi l'échelle séquentielle (phrase finale).

Cette manière de jongler entre les deux langues est caractéristique pour les quatre radios étudiées. Un auditeur uniquement francophone peut ainsi suivre les émissions, mais seuls les auditeurs bilingues posséderont la totalité des informations.

Une minorité d'émissions de Radio Gazelle cependant ne suit pas ce schéma ; l'arabe st dominant.

Radio JM a moins d'émissions bilingues. Par contre l'utilisation de salutations et formules en hébreu est courante. Cette pratique linguistique est également employée par de nombreux animateurs et animatrices des trois autres radios. Si l'on se réfère à nos entretiens, il apparaît que plusieurs animateurs ne parlent pas la langue étrangère en question. L'utilisation ponctuelle de mots en langue « d'origine » ne relève donc pas d'un manque de vocabulaire en français, mais doit remplir une autre fonction. Il semble que ces mots ont une valeur de signal, plutôt que de communiquer une appartenance commune.

30 Un autre trait marquant est l'accent des animateurs. Dans les médias audiovisuels nationaux et locaux, il est rare d'entendre un accent étranger émanant d'un présentateur tandis que dans les émissions «communautaires » cette caractéristique représente la norme plutôt que l'exception.

31 La langue parlée constitue un mécanisme concret d'inclusion/exclusion par rapport à un champ social. Les émissions bilingues permettent aux auditeurs qui ne possèdent pas un bon niveau de français de suivre le programme. Le bilinguisme a cependant une signification qui va au-delà de cet aspect purement «technique». La langue ne constitue pas seulement un "support » pour la communication; comme nous l'avons évoqué ci-dessus elle implique et expose certaines compétences sociales. Grâce à ces 
caractéristiques, une langue peut dans sa globalité devenir un symbole d'identité collective (Fishman, 1989 : 32).

Vues dans cette perspective, les émissions bilingues, l'utilisation de certains mots dans les langues d'origine et une prononciation non conforme (l'accent) de nombreux animateurs véhiculent une identité collective. En même temps le français est largement utilisé. Enfin, la radio, en tant que média, semble structurer le rythme et le style linguistique au-delà des différences de langue : un ton monologique, sérieux pour la présentation d'actualités, une forme dialogique partiellement simulée pour les émissions de jeunesse. Les émissions créent ainsi une expression linguistique particulière qui mélange les éléments symboliquement légitimes et les éléments qui sont symboliquement dévalorisés dans d'autres médias français. Cette forme "audiolinguistique " n'est possible que parce que les radios communiquent par la langue parlée.

Participation à distance et à proximité

33 Les médias permettent une participation qui ne nécessite pas de présence physique. Dans son ouvrage, Anderson (1983) montre que ce n'est qu'avec la généralisation de l'impression des livres et des journaux qu'il est devenu possible de créer un imaginaire collectif de contemporanéité, et par là même de communauté, entre personnes géographiquement éloignées. La communication d'informations, de publicités, de discussions etc. dans les émissions communautaires va dans le même sens, elle renvoie à l'appartenance.

Une partie importante de la communication concerne les pays d'origine des auditeurs, soit dans le cas de Radio JM: Israël. À travers la transmission et la rédaction d'actualités, les radios s'inscrivent dans un espace transnational où les " diasporas » à Marseille participent activement à la vie politique dans les pays d'origine. Cette forme de participation n'est pas inconnue par les différentes autorités politiques des pays en question. Dans certains cas, les radios font l'objet d'interventions de nature stratégique de la part des acteurs politiques étrangers. Par exemple, durant l'enquête, l'émission comorienne de Radio Galère a reçu la visite d'un haut fonctionnaire comorien qui souhaitait s'adresser à la communauté sur les ondes marseillaises. Sur JM, les personnalités israéliennes interviewées dans les reportages démontraient régulièrement leur volonté de mobiliser les auditeurs marseillais en les sensibilisant au contexte israélien. Cependant ces formes de participation politique plus au moins manifestes ne sont pas les seuls liens d'appartenance et d'identification au pays d'origine. Ceux-ci sont également d'ordre pratique (informations qui facilitent l'organisation de voyages, l'envoi d'objets, le transfert d'argent, les appels téléphoniques), et culturel (informations sur la littérature, la musique, le cinéma etc. du pays en question).

Localement, les radios comme Radio Gazelle et Radio JM permettent la participation à une pratique religieuse, respectivement musulmane et juive. Et un grand nombre d'informations, de publicités et de sujets de discussion dans les quatre radios renvoient à un tissu commercial et associatif "communautaire». Les liens transnationaux et religieux sont à la base des pratiques locales comme le commerce de viande halal et kacher et la création d'associations comoriennes, italiennes, juives... Les activités sont diverses comme le montre l'exemple des annonces explicitement nommées « communautaires » de Radio JM : 
«La synagogue Mazeltov de Bonneveine vous informe qu'elle organise son loto annuel le dimanche 5 mars (...)».

"Autre information communautaire, il s'agit de l'association des parents d'élèves de l'école Yavné qui organise le 11 mars une soirée orientale... ».

«La chorale hébraïque Renanim Marseille dont le répertoire se compose de chants traditionnels en hébreu, Yiddish et Ladino vous informe qu'elle recrute des voix d'homme..." ". prennent leur signification par rapport au contexte français ou marseillais. L'identité «maghrébine », qui ne serait pas, nécessairement, socialement pertinente au Maghreb, apparaît dans les publicités de Radio Gazelle comme la base d'intérêts de consommation commerciale partagés par une certaine catégorie de personnes :

«Optique International, 23 rue d'Aix, le moins cher des Bouches-du-Rhône, vous propose un grand choix de marques à prix d'usine et (...).Waïba se fera un plaisir d'accueillir la clientèle maghrébine».

«Pour tous vos envois à destination du Maghreb la société Solifex vous propose ses services... ».

Ainsi, la notion de communauté semble à première vue ancrée dans des appartenances et des pratiques qui établissent des frontières sociales assez claires. Cependant les quatre radios diffusent de nombreux énoncés et informations concernant l'espace local qui n'y font pas référence. La politique locale, le sport, les manifestations culturelles municipales et régionales sont relayés avec les mêmes implications d'appartenance que les informations « communautaires » et celles qui concernent les pays d'origine. Toutes les radios s'appuient également sur les médias nationaux français et transmettent en grande partie la même « actualité » quotidienne. Enfin les quatre radios ont également des émissions de conseils juridiques, médicaux et sociaux. Certaines émissions, notamment de Radio Gazelle et Radio Galère, sont même le moyen privilégié d'accès à ces informations pour des groupes sociaux affectés par l'analphabétisme par exemple ou par le contact limité avec les espaces publics. Ainsi les émissions juridiques de Radio Gazelle s'adressent largement à un public féminin d'origine maghrébine qui obtient ici des informations sur le droit familial. Les émissions comoriennes bilingues semblent atteindre un public récemment installé qui n'a pas les compétences pour accéder aux médias écrits et francophones.

La communication d'identité collective est simultanément une grille de lecture pour des pratiques concrètes et le résultat de celles-ci. L'analyse nous montre que cet imaginaire et ces pratiques se déploient dans un espace social fragmenté et pluriel, à la fois transnational et local. Les formes de participation que véhiculent les radios communautaires sont multiples et reflètent la complexité et l'hétérogénéité des notions de communauté.

Appartenances multiples

39 Afin d'explorer cette complexité une analyse sémantique des discours s'avère utile. À partir de l'analyse codée des entretiens et des émissions enregistrées, on peut identifier trois noyaux sémantiques autour desquels sont construits les discours des animateurs et des auditeurs.

Le premier noyau sémantique concerne la notion de « communauté » qui est associée à l'idée d'une origine commune et elle apparait comme innée. Dans l'expression concrète, cette notion renvoie à la religion, à l'origine nationale ou régionale.

Revue européenne des migrations internationales, vol. 19 - $n^{\circ} 2$ | 2003 
« La communauté... c'est la religion, je crois. C'est ça la base » (entretien animateur, Radio JM).

"Quelqu'un qui est né musulman, il va pas se cacher [...] et on sait tous, toute la communauté, que ce projet il traîne « (émission, Radio Gazelle).

« Nous émettons vers la communauté juive, la radio fait partie de la communauté » (entretien animateur, Radio JM).

41 d'appartenance et comme communauté politique. L'appartenance est ici exprimée à travers l'idée de « citoyenneté » et de participation civile et politique.

" Ça nous concerne tous, en tant que citoyens, c'est un débat très important et nous avons tous quelque chose à dire là-dessus » (émission, Radio Gazelle).

42 Leisième noyau sémantique concerne Marseille comme cadre d'identité commune. L'idée de Marseille comme « ville multiculturelle » et « multi-communautaire » est mise en avant. Elle exprime une singularité par rapport au reste de la France.

«C'est très français, cette idée, il y a le big boss qui décide tout, monsieur le président où je sais, moi... Ici, au moins, à Marseille on n'est pas trop comme ça, c'est pas comme ailleurs en France » (émission, Radio Gazelle).

«La cité phocéenne, la ville multi-communautaire où nous avons tous une place, qui voudrait changer ça ?» (émission, Radio Galère).

On remarquera que le discours "communautaire", dont l'une des prémisses est la nature clairement délimitée de la "communauté », n'est pas contesté ou remis en question par le chevauchement effectif, au niveau pratique, des catégories telles que par exemple " maghrébin », « algérien », « musulman » ou " kabyle ». Cela indique déjà la nature flexible des catégories sémantiques, il ne s'agit pas ici d'une articulation logique mais d'une stratégie pragmatique. En effet, les radios combinent les trois discours dans une expression inter-discursive particulière. Ainsi les idées d'une communauté ethnique et d'une communauté républicaine politique ne sont pas séparées, mais servent au contraire de points de repère combinés dans les énoncés sur l'appartenance et l'identité collective. Les contradictions apparentes n'apparaissent pas comme telles :

«Un Algérien, enfin qui est né en France d'origine algérienne, il est français, parce que quelqu'un qui est né en France il est automatiquement français, il n'appartient pas à la communauté algérienne, il appartient à la communauté française. [...] Il faut jamais oublier ses origines. Moi, je suis resté algérien. Les jeunes aussi, ils sont algériens, mais ils sont d'abord français » (émission, Radio Gazelle).

"Je suis peut-être comorien dans l'âme, mais j'ai la mentalité d'ici » (entretien animateur, Radio Gazelle).

La «frontière" sémantique la plus clairement établie se trouve entre le discours «marseillais » et le discours sur la République française; la différence entre "nous marseillais et « eux », le reste de la France, est régulièrement thématisée. Il ne s'agit pas là d'interpréter la « communauté » marseillaise en termes d'ethnicité. Les adjectifs qualificatifs employés pour caractériser cette « communauté » portent justement sur la nature "plurielle» et "multi-communautaire» de la ville. Encore une fois, la dimension ethnique n'est pas problématisée, mais est au contraire intégrée aux autres critères d'appartenance.

Ainsi, les émissions communautaires combinent des notions sémantiques qui circulent dans un espace discursif public plus large et forment un cadre souple et inclusif de l'identité culturelle collective. La dimension ethnique est présente, mais elle n'est pas

Revue européenne des migrations internationales, vol. $19-n^{\circ} 2$ | 2003 
exclusive et ne relève pas d'une notion de fermeture. Au contraire, elle se combine avec les sentiments d'appartenance républicaine et locale.

Communautés et musiques

Les styles musicaux des émissions rejoignent de manière complémentaire ces processus sémantiques. La musique est une forme sonore en mouvement, mais elle est aussi un phénomène social qui peut évoquer des ambiances, des sentiments et des identités individuelles et collectives. Elle circule dans le monde comme produit commercial et comme pratique artistique (Appadurai, $1988 ; 1990$ ), mais, dans ce processus, elle se voit attribuer des significations variées dans des espaces sociaux concrets. Des formes musicales locales deviennent accessibles au niveau transnational, des formes transnationales prennent des significations particulières dans l'espace local (Hannerz, 1989).

Les radios communautaires constituent des espaces sociaux où les formes musicales prennent très souvent leur signification par rapport à l'idée d'une identité collective. Il est alors pertinent de distinguer les émissions qui s'adressent à un public plus âgé de celles qui s'adressent à un public jeune.

Nous avons mentionné qu'une partie de la communication a comme référence le pays d'origine, soit comme l'arène de pratiques concrètes, soit comme notion sémantique. La première catégorie d'émissions est caractérisée par une communication musicale qui lui fait référence. Les émissions de Radio Gazelle, de musique classique orientale, celles de Radio Diva et de Radio Galère, de musique italienne des années 50 et celles de JM, de musique judéo-arabe, pour ne mentionner que quelques exemples, sont toutes assez homogènes sur le plan stylistique. La musique est composée et produite dans une région géographique bien délimitée et date d'une période également déterminée. Le discours qui accompagne ces genres musicaux a souvent une dimension nostalgique, orientée vers le passé.

Les émissions qui s'adressent aux jeunes sont caractérisées par une plus grande hétérogénéité stylistique. Il s'agit de genres musicaux qui font partie de réseaux de distribution transnationaux bien établis, comme le pop, le rock, le rap, le reggae, la salsa et le raï. Bien qu'il y ait des différences entre les émissions, on retrouve ces musiques dans les quatre radios. Cela permet d'ailleurs une observation intéressante concernant les discours autour de cette communication musicale. Certains des genres mentionnés ci-dessus sont effectivement associés par les auditeurs et les animateurs à la notion d'un espace transnational partagé. Ainsi la salsa et le zouk sont décrits comme étant "pour tout le monde ». Par contre le rap est présenté par certains auditeurs et animateurs d'origine maghrébine comme «notre » musique tandis qu'un animateur de Radio Diva souligne que le rap «ce n'est pas pour nous». D'autres animateurs, par exemple de Radio JM, mais également de Gazelle et Galère ont associé le rap à un style de vie jeune, et certains ont évoqué un enracinement particulier du rap à Marseille qui confèrerait à ce genre une identité locale.

50 Le raï, forme musicale qui s'est imposée largement, joué dans des cabarets oranais mais aussi dans des soirées intellectuelles parisiennes (Schade-Poulsen,1997), est également l'objet d'une interprétation qui varie d'une station radio à une autre. Classée comme « musique du monde » sur Radio Galère et Radio JM elle devient « de chez nous » sur Radio Gazelle.

51 Paradoxalement c'est donc dans les émissions s'adressant aux jeunes que l'on trouve les discours les plus explicites sur la musique comme aspect d'une identité collective 
culturelle. Les genres musicaux, largement globalisés, qui font partie de l'espace des goûts des jeunes, sont réinterprétés dans le cadre des radios et se voient attribués des significations qui renvoient tantôt à l'enracinement local tantôt à l'origine «communautaire ». Les émissions musicales s'adressant à un public plus âgé, sont plutôt accompagnées d'un discours de nature nostalgique qui ne thématise pas l'aspect identitaire de la musique ${ }^{7}$.

52 Ainsi la musique permet aux auditeurs et aux animateurs de déployer une pratique communicative particulière qui rejoint et complète la construction polysémique discursive de l'identité collective.

Conclusion

Quelle est donc la notion de « communauté » qui apparaît sur les ondes marseillaises? La communication des radios « communautaires » s'oriente-t-elle vers une fermeture identitaire?

La création et l'existence même des radios ne peuvent être comprises que par rapport à la problématique plus large de représentation médiatique d'une identité commune. Les auditeurs interviewés disent tous "se reconnaître " dans les émissions des quatre radios. De plus, animateurs et auditeurs partagent le sentiment d'une sousreprésentation de « leur culture » dans les médias nationaux français.

55 Cependant l'analyse montre bien que ce serait une erreur de comprendre la communication "communautaire" comme culturellement homogène et comme opposée à la société française plus largement. À l'inverse c'est justement à travers la construction d'un "nous ", incluant la dimension ethnique et communautaire, sans en faire une « frontière » absolue - une construction, qui traverse les différents aspects de la communication - que les radios facilitent la participation et l'identification au contexte français et à la vie locale.

56 Les quatre radios ont en commun une communication qui mêle les notions d'appartenance dans une expression d'identité collective culturelle hybride. Vue dans un contexte plus large, l'analyse des radios illustre que l'identité « communautaire » ne s'oppose pas nécessairement à l'appartenance à la "République », mais que, bien au contraire, l'identité collective telle qu'elle est construite et négociée en pratique, peut réconcilier les différents points d'attachement dans une appropriation qui, dans le cas présent, passe par la référence locale.

57 Les développements futurs des radios communautaires sont liés aux changements technologiques qui permettent, via le satellite, le câble et Internet, d'accéder directement aux médias du " pays d'origine $»^{8}$. Ainsi depuis quelques années les radios mettent en place des stratégies de regroupements et parfois même des collaborations internationales 9 . Mais, les processus plus larges, qui forment la construction et la négociation sociales d'identité collective et qui établissent des "frontières " sociales rigides ou souples, sont plus importants pour les pratiques des animateurs et des auditeurs des radios et pour leur reconnaissance et l'identification à tel ou tel média. 


\section{BIBLIOGRAPHIE}

ABDALLAH Mogniss H. (2001) Médias et immigration. Dynamique interassociative sur les ondes, Hommes \& Migrations, 1230, mars-avril 2001.

AMIT Vered (2002) Reconceptualizing community, in Vered Amit Éd., Realizing Community, London \& New York, Routledge, pp. 1-20.

ANDERSON Benedict (1983) Imagined Communities, Verso, London \& New York.

APPADURAI Arjun (1988) Introduction, in Arjun Appadurai Éd., The social life of things, Cambridge, Cambridge University Press.

APPADURAI Arjun (1990) Disjuncture and Difference in the Global Cultural Economy, in M. Featherstone Éd., Global culture, London, Sage.

BARTH Fredrik (1969) Introduction, in Fredrik Barth Éd., Ethnic Groups and Boundaries, London, George Allen and Unwin.

BOURDIEU Pierre (1982) Ce que parler veut dire, Paris, Fayard.

CHEVAL Jean-Jacques (1997) Les radios en France, Paris, Éditions Apogée.

COHEN Anthony P. (1994) Boundaries of consciousness, consciousness of boundaries. Critical questions for anthropology, in Hans Vermeulen \& Cora Govers Éds., The Anthropology of Ethnicity. Beyond Ethnic Groups and Boundaries, Amsterdam, Het Spinhuis, pp. 59-80.

FISHMAN Joshua A. (1989) Language and Ethnicity, in Minority Sociolinguistic Perspective, Clevedon \& Philadelphia, Multilingual Matters LTD.

HALL Stuart ; HOBSON Dorothy ; LOWE Andrew \& WILLIS Paul Éds (1980) Culture, media, language, London, Hutchinson.

HANNERZ Ulf (1989) Culture Between Center and Periphery : Toward a Macro anthropology, Ethnos, 54, pp. 200-216.

HERTZFELD Michael (1997) Cultural Intimacy: Social Poetics in the Nationstate, London \& New York, Routledge.

LULL James (1995) Media, Communication, Culture, Cambridge, Polity Press.

MOUFFE Chantal (1993) The Return of the Political, London, Verso.

SCHADE-POULSEN Marc (1997) Which world ? On the diffusion of Algerian raï to the West, in Kirsten Hastrup and Karen Fog Olwig Éds., Siting Culture, London, Routledge pp. 59-85.

VANOYE Francis (1979) Récit écrit, récit filmique, Paris, CEDIC.

WICKER Hans-Rudolf (1997) Introduction. Theorizing Ethnicity and Nationalism, in Hans-Rudolf Wicker Éd., Rethinking Nationalism and Ethnicity. The Struggle for Meaning and Order in Europe, Oxford \& New York, Berg, pp. 1-42.

\section{NOTES}

1. Il existe d'autres radios communautaires dans la région, comme Radio Soleil, qui est une antenne d'une radio nationale et dont la dimension locale est plus limitée. 
2. Pour une description détaillée de l'histoire des radios libres voir Cheval (1997).

3. On considère le médium écrit comme monodique, le cinéma et la télévision sont polyphoniques ; ils combinent plusieurs matières d'expression. La radio pourrait, dans cette optique être considérée comme un médium à polyphonie restreinte. Voir aussi Vanoye (1979).

4. L'observation participante permet de partager une expérience sociale avec les informateurs. Cette expérience peut révéler des aspects de l'univers social qui ne sont pas exprimés dans la présentation discursive de celui-ci. Certains théoriciens veulent voir une distinction implicite entre l'observation participante et les données d'origine discursive. Il peut donc sembler étonnant, de prime abord, d'utiliser l'observation participante pour l'étude d'une pratique qui est avant tout discursive. Cependant, une distinction rigide entre les pratiques discursives et non-discursives s'avère peu fructueuse. Les énoncés sont des actes entrepris dans un univers physique ; les pratiques non-verbales prennent sens à travers la pratique discursive. Même si la communication radiophonique crée un espace social qui n'est pas attaché à un lieu, cette communication prend forme à travers des pratiques qui se déroulent dans un espace physique et observable.

5. Il convient de noter que l'auteur de ces pages n'avait pas de relation avec les radios étudiées avant d'arriver (de Copenhague) à Marseille pour conduire ce travail. L'accès aux radios s'est fait sans difficulté ; les studios étaient en effet caractérisés par un vaet-vient important qui a facilité le contact avec les informateurs et l'observation participante.

À différence des entretiens, une grande partie du matériel analysé consiste en des données qui n'ont pas été influencées par notre présence ; celles-ci dépendant de la communication radiophonique.

6. Des discussions théoriques ont fait de cette distinction une question herméneutique concernant la nature, soit attribuée, soit choisie de l'identité collective. Ainsi Anthony P. Cohen (1994: 76) défini le groupe ethnique comme : « an aggregate of selves each of whom produces ethnicity for itself ». Cependant, nous ne percevons pas cette dualité comme une dichotomie, mais comme une complémentarité - la construction d'identité collective culturelle implique des processus de classification et d'attribution tant externes qu'internes.

7. La séparation analytique des auditeurs en deux groupes selon l'âge peut sembler réductrice par rapport à la complexité du champ. Les émissions atteignent parfois un autre public que celui auquel elles s'adressent explicitement. L'intérêt renouvelé de la part de certains jeunes auditeurs pour des genres musicaux « classiques » en est un exemple. Cependant ces pratiques font elles aussi le plus souvent partie de la dimension identitaire de la consommation musicale.

8. Les progrès technologiques peuvent également servir ou modifier la communication radiophonique. Certaines radios ont ainsi crée leur site Internet.

9. Pour une discussion de ces stratégies voir Abdallah (2001). 


\section{AUTEUR}

\section{KATRINE RøMHILD BENKAABA}

Doctorante à l'IEP, Aix-en-Provence. 